\title{
Size-tunable electroluminescence characteristics of quantum confined Si nanocrystals embedded in Si-rich oxide matrix
}

Cite as: Appl. Phys. Lett. 116, 231105 (2020); doi: 10.1063/5.0001840

Submitted: 20 January 2020 - Accepted: 2 June 2020 .

Published Online: 12 June 2020
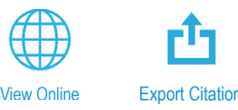

Arijit Sarkar, (DD Rajshekhar Bar, ${ }^{2,3}$ Sudarshan Singh, ${ }^{2}$ Rup Kumar Chowdhury, ${ }^{2}$ Sekhar Bhattacharya, ${ }^{4}$ Amal Kumar Das, ${ }^{2}$ and Samit K. Ray ${ }^{2,5, a)}$ (D)

\author{
AFFILIATIONS \\ ${ }^{7}$ Advanced Technology Development Center, Indian Institute of Technology, Kharagpur-721302, India \\ ${ }^{2}$ Department of Physics, Indian Institute of Technology, Kharagpur-721302, India \\ ${ }^{3}$ Midnapore College, Midnapore-721101, India \\ ${ }^{4}$ Core Laboratories, King Abdullah University of Science and Technology, 23955-6900 Saudi Arabia \\ ${ }^{5}$ S.N. Bose National Center for Basic Sciences, Sector-III, Salt Lake, Kolkata-700098, India \\ ${ }^{a)}$ Author to whom correspondence should be addressed: physkr@phy.iitkgp.ac.in
}

\begin{abstract}
Tunable electroluminescence properties of size-controlled Si nanocrystals embedded in silicon rich oxide films are demonstrated at room temperature, using an active light emitting layer in the metal oxide semiconductor device structure. Plasma enhanced chemical vapor deposited Si-rich oxide films were annealed at elevated temperatures to form Si nanocrystals of varying diameters. A typical redshift in the photoluminescence peak is observed with increasing annealing temperature, confirming the formation of quantum confined Si nanocrystals. The carrier transport and light emission mechanism have been studied in detail through current-voltage characteristics and ultrafast transient spectroscopy, respectively. The origin of electroluminescence and the size-tunable emission peak have been analyzed and attributed to the radiative recombination of carriers within Si nanocrystal quantum wells. The fabricated Si nanocrystal-based metal oxide semiconductor light emitting diode and the resultant size-dependent tunable electroluminescence are very attractive as a potential CMOS compatible optical source for future photonic integrated chips.
\end{abstract}

Published under license by AIP Publishing. https://doi.org/10.1063/5.0001840

A great deal of research effort has been dedicated to develop CMOS compatible optical sources on Si platforms replacing hybrid systems based on $\mathrm{GaN}$, GaAs, and $\mathrm{Ge}$ on $\mathrm{Si}^{1-4}$ Reducing the dimensions of $\mathrm{Si}$ structures below the excitonic Bohr radius of $\mathrm{Si}(\sim 4.8 \mathrm{~nm})$ results in light emission due to the quantum confinement effect (QCE). ${ }^{5-8}$ Different approaches of light emission through the QCE have been reported to date based on Si nanowires, ${ }^{9-12}$ nanocones, ${ }^{13,14}$ colloidal nanocrystals, ${ }^{15,16}$ and nanocrystals in the oxide matrix. ${ }^{5,17-19}$ Among them, Si nanocrystals ( $\mathrm{Si} \mathrm{NCs}$ ) embedded into the $\mathrm{SiO}_{2}$ matrix are most promising due to chemical stability and wavelength selective size-tunable photoluminescence $(\mathrm{PL})$. $^{8,17,20-22}$

Although there are many reports of size-tunable PL emissions from Si NCs involving the QCE, their utilization as Si-based light emitting diodes (LEDs) is few. The demonstrations of electroluminescence (EL) are mostly broad, covering the visible spectral range or defectrelated blue emissions only. ${ }^{1,23-26}$ López-Vidrier et al. ${ }^{27}$ and Fu et al. ${ }^{6}$ have recently demonstrated efficient EL in the $\mathrm{Si} \mathrm{NC/SiO}$ superlattice structure, but they too observed EL over a broad spectral range. Therefore, the demonstration of tunable EL emission utilizing the QCE in Si NCs may provide a colossal step toward optoelectronics in $\mathrm{Si}$ platforms. We report unique size-tunable electroluminescence characteristics at room temperature from Si NCs embedded into the $\mathrm{SiO}_{2}$ matrix. The injected current transport through the devices has been analyzed. The detailed analysis revealed that the QCE in Si NCs leads to size-tunable PL and EL emission.

Si NCs of different diameters embedded into the $\mathrm{SiO}_{2}$ matrix were fabricated by depositing silicon rich oxide (SRO) films using the plasma enhanced chemical vapor deposition (PECVD) technique followed by annealing of those films at three different temperatures $\left(900^{\circ} \mathrm{C}, 1000^{\circ} \mathrm{C}\right.$, and $\left.1060^{\circ} \mathrm{C}\right)$. Henceforth, the samples annealed at $900{ }^{\circ} \mathrm{C}, 1000^{\circ} \mathrm{C}$, and $1060^{\circ} \mathrm{C}$ are referred to as $\mathrm{P} 900, \mathrm{P} 1000$, and $\mathrm{P} 1060$, respectively. The details of the device fabrication, instruments 
for material characterization, and measurement processes are provided in the supplementary material. High-resolution TEM (HRTEM) micrographs presented in Figs. 1(a)-1(c) confirm the formation of distinct spherical Si NCs with different average sizes embedded uniformly within the SRO matrix for P900, P1000, and P1060 samples. For all the samples, the densities of smaller diameter NCs are found to be higher along with large size NCs. It has been found out that the P900 sample has the average size of $\sim 3 \mathrm{~nm}$, while for P1000 and $\mathrm{P} 1060$, the average sizes are $\sim 4.5 \mathrm{~nm}$ and $\sim 6.5 \mathrm{~nm}$, respectively. Histograms showing the size distribution of Si NCs for all the samples are presented in the supplementary material in Fig. S2. On annealing at higher temperatures, the available energy allows the excess Si atoms in the SRO matrix to diffuse, forming the Si NCs through the Ostwald ripening process. ${ }^{5,27-29}$ The observed average size of the NCs is comparable to the excitonic Bohr radius of Si, suggesting that QCE may play a dominating role in tuning the optical properties of samples. ${ }^{5} \mathrm{~A}$ magnified image of a single Si NC, revealing lattice fringes with different orientations and suggesting the polycrystalline nature of the grown $\mathrm{Si} \mathrm{NCs}$, is presented in the supplementary material. The polycrystalline nature and chemical stoichiometry of the Si NCs were further analyzed using XRD and XPS data, respectively, and are discussed in detail in the supplementary material.

Normalized PL spectra of the as-grown SRO film (un-annealed), P900, P1000, and P1060 at room temperature are co-plotted in Fig. 1(d). The PL emission peak exhibits a gradual redshift with increasing annealing temperature of the SRO films. The as-prepared SRO film has weak emission intensity with a peak centered at $\sim 440 \mathrm{~nm}$. This emission is frequently ascribed to oxygen-related defect states in the $\mathrm{SiO}_{2}$ matrix $\left.(\mathrm{O}-\mathrm{Si}-\mathrm{O} / \mathrm{Si}=\mathrm{O})\right)^{2,19,20,30}$ For P900, P1000, and $\mathrm{P} 1060$ samples annealed at different temperatures, the PL peak is centered at 560,594 , and $642 \mathrm{~nm}$, respectively. It is to be noted that a small hump at $\sim 440 \mathrm{~nm}$ is also observed for the P900 sample, which is absent in
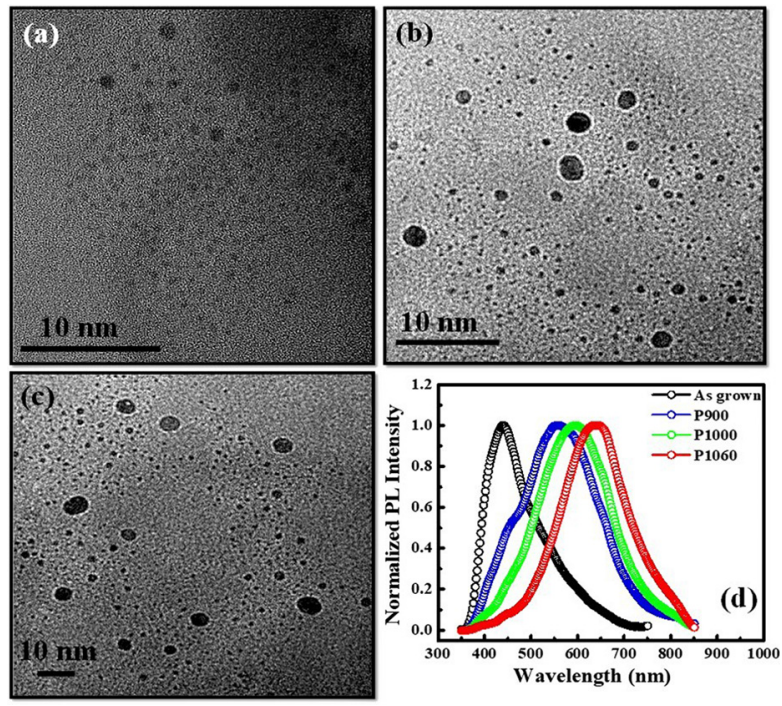

FIG. 1. Typical plane-view HRTEM images of Si NCs of different sizes embedded into the SRO matrix for (a) P900, (b) P1000, and (c) P1060 samples. (d) Normalized room temperature PL spectra of as-grown and SRO samples annealed at different temperatures $\left(900^{\circ} \mathrm{C}, 1000^{\circ} \mathrm{C}\right.$, and $\left.1060^{\circ} \mathrm{C}\right)$. samples annealed at higher temperatures. This signifies the reduction of defect state density with increasing annealing temperature. An enhanced recrystallization on annealing at high temperature results in 10 -fold intense PL emission due to NCs compared to the defectrelated emission. The monotonous redshift of the PL peak as a function of temperature suggests that the emission is due to excitonic recombination as a result of QCE of carriers within Si NCs. ${ }^{2,19,20}$ Due to the QCE, radiative recombination occurs between degenerate states in the $\Delta$-valley and the $\Gamma$-point, resulting in strong PL emission. ${ }^{2,19,20,30}$ The observed PL peaks are Gaussian in shape, and broadening of the emission peak is due to the overall size distribution of the NCs in the corresponding samples.

To gain a proper insight into the carrier dynamics involved with the PL characteristics of Si NCs, time-resolved photoluminescence (TRPL) measurements at the PL peak wavelengths have been performed by employing time-correlated photon counting spectroscopy. The corresponding PL decay spectra at room temperature are presented in Fig. 2. Time-resolved PL spectra have been fitted with the standard stretched exponential decay function widely used for this kind of system to find out the effective carrier lifetime $(\tau)$. For P900, two characteristic relaxation times are found to be $\tau_{1}=0.82 \mu \mathrm{s}$ and $\tau_{2}=4.51 \mu \mathrm{s}$. The corresponding values of $\tau_{1}=0.86 \mu$ s and $\tau_{2}=4.92$ $\mu$ s are estimated for the P1000 sample, whereas for the P1060 sample, the estimated values of lifetimes are $\tau_{1}=0.98 \mu \mathrm{s}$ and $\tau_{2}=5.58 \mu \mathrm{s}$. The higher carrier lifetime $\left(\tau_{2}\right)$ of samples varies from 4.51 to $5.58 \mathrm{~ms}$, which is much larger than the defect-induced recombination lifetime typically on the order of nanoseconds. So the origin of PL emissions is attributed to the quantum confinement effect in Si NCs in agreement with the results reported by several groups. ${ }^{1,6,11,18,26}$ On the other hand, the observation of a faster decay channel $\left(\tau_{1}\right)$ may be due to the presence of interfacial defects between $\mathrm{Si}$ NCs and the $\mathrm{SiO}_{2}$ matrix surrounding it. For further studies of defect-induced carrier dynamics in detail, transient reflectivity $(\Delta \mathrm{R})$ measurements at an ultrafast timescale were carried out for a typical sample (P1000). The experimental details and results are presented in the supplementary material in Sec. S5 and Fig. S4, showing the ultrafast depopulation of carriers from quantized states of Si nanocrystals on the pico-second timescale to the non-radiative trapping states.

To study the charge transport behavior of the fabricated metaloxide-semiconductor light emitting diode (MOSLED) based on $\mathrm{Si}$ NCs embedded into the SRO matrix, current-voltage (I-V)

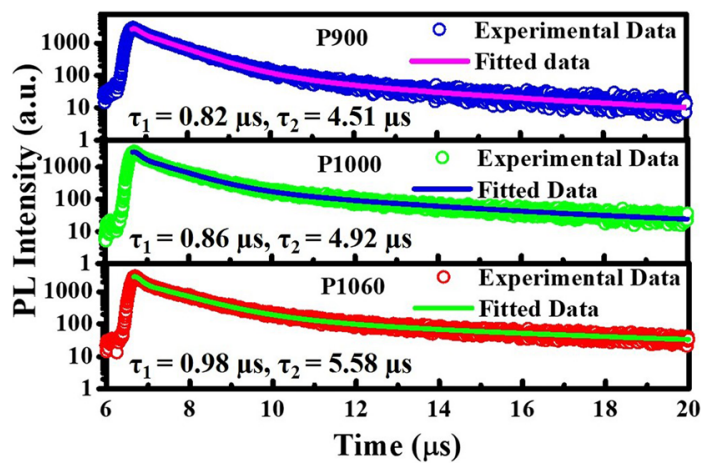

FIG. 2. Time-resolved photoluminescence decay spectra of P900, P1000, and P1060 samples (from top to bottom). 
characteristics of the P1060 device under the forward bias condition have been measured and are presented in Fig. 3(a). The device shows a MOS-like I-V behavior with a forward current of $7 \mathrm{~mA}$ at an applied forward bias of $10 \mathrm{~V}$ with a turn-on voltage of $2 \mathrm{~V}$. The schematic diagram of the device is presented in the inset of Fig. 3(a). To investigate the charge injection mechanism, which can be either direct or Fowler-Nordheim (F-N) tunneling, the current density $(\mathrm{J})$ and electric field (E) determined from the I-V data are presented as $\ln \left(\mathrm{J} / \mathrm{E}^{2}\right)$ as a function of (1/E) plot in Fig. 3(b). The F-N tunneling can be represented analytically by

$$
J_{F N}=\frac{A}{4 \phi_{B}} E^{2} \cdot \exp \left(-\frac{2 B \phi_{B}^{3 / 2}}{3 E}\right),
$$

where $A$ and $B$ are the constants and $\phi_{B}$ is the effective barrier height for carrier injection. ${ }^{1,6,31}$ The linear fitted F-N plot in the high electric field region reveals that the carrier conduction in the fabricated device is dominated by the F-N tunneling mechanism between the NCs through the triangular-shaped $\mathrm{SiO}_{2}$ barriers. ${ }^{1,6,31}$ In a low electric field region below $4.38 \times 10^{6} \mathrm{~V} / \mathrm{cm}$, the $\mathrm{F}-\mathrm{N}$ plot becomes non-linear, denoting that the carrier conduction between NCs follows the direct tunneling mechanism through $\mathrm{SiO}_{2}$ barriers. This kind of carrier transport mechanism combining both $\mathrm{F}-\mathrm{N}$ tunneling and direct tunneling in different electric field regions has been reported in the literature for similar systems. ${ }^{6,18,31,32}$

Each of the MOSLEDs fabricated using P900, P1000, and P1060 samples exhibited EL emission of different colors at room temperature, which is visible even in the naked eye. Figures 4(a) and 4(b) present the deconvoluted EL spectra from P900 and P1000 devices, respectively. The spectra were obtained at forward bias conditions when current densities of $3.5 \mathrm{~mA} / \mathrm{cm}^{2}$ and $4.4 \mathrm{~mA} / \mathrm{cm}^{2}$ were passed through P900 and P1000 samples, respectively. Interestingly, the broad EL spectra for both devices can be deconvoluted into two distinct peaks. For the P900 sample, the emission is in the range of 360-730 nm, with peaks centered at $\sim 445$ and $\sim 567 \mathrm{~nm}$, whereas the emission is in the range of $360-760 \mathrm{~nm}$ with peaks centered at $\sim 443$ and $\sim 603 \mathrm{~nm}$ for the P1000 sample. From the previous discussion of PL data [Fig. 1(d)], it can be deduced that the peaks at $\sim 445$ and $\sim 443 \mathrm{~nm}$ are due to the radiative transitions associated with oxygen defect states in $\mathrm{SiO}_{2}$. However, the emission centered at $\sim 567 \mathrm{~nm}$ for P900 and $\sim 603 \mathrm{~nm}$ for P1000 samples is at similar energy in agreement with corresponding
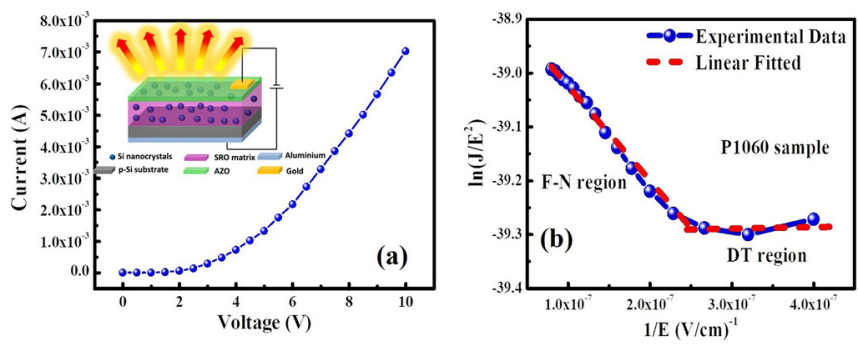

FIG. 3. (a) Current-voltage (I-V) characteristics in the forward bias condition of the fabricated Si NC-based MOSLED device (P1060), while the inset shows the schematic illustration of the same. (b) The plot of $\ln \left(J / E^{2}\right)$ as a function of $1 / E$ obtained from the I-V plot fitted using the Fowler-Nordheim equation at a higher field and direct tunneling one at a lower field.
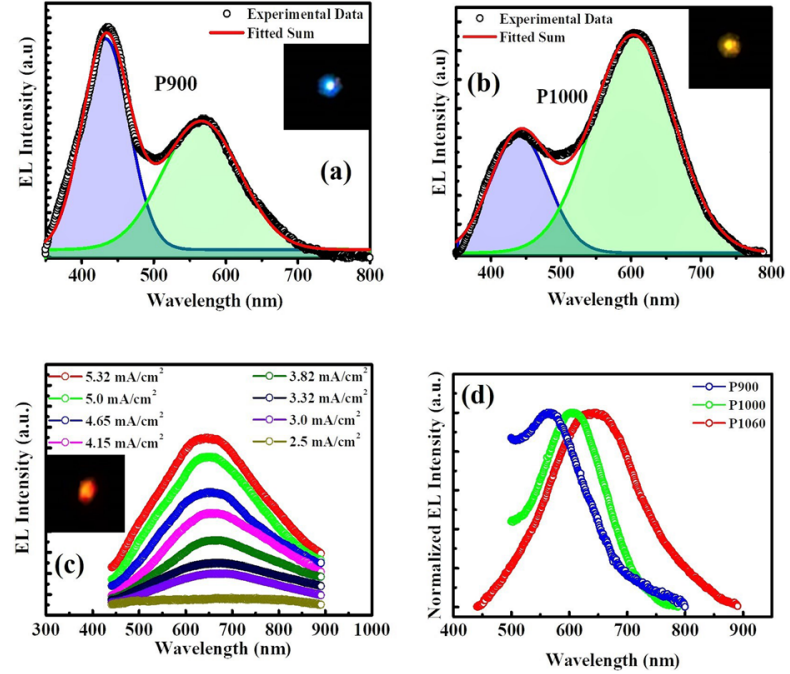

FIG. 4. EL spectrum of (a) P900 sample at an injection current density of $3.5 \mathrm{~mA} / \mathrm{cm}^{2}$ and (b) P1000 sample at $4.4 \mathrm{~mA} / \mathrm{cm}^{2}$. The insets show the photographs of the EL emissions from the respective samples. (c) EL spectra of the P1060 sample as a function of injected current density, with the inset showing the photograph of the strong emission at $5.32 \mathrm{~mA} / \mathrm{cm}^{2}$. (d) Normalized EL spectra of P900, P1000, and P1060 samples considering the contribution from quantum-confined emission in NCs only.

PL data [Fig. 1(d)], suggesting that the origin of those emissions is due to the QCE of carriers in Si NCs. The defect-related peak is intense compared to QCE-related emission for the P900 sample [Fig. 4(a)], whereas an opposite result is observed for the P1000 sample, where QCE-related emission is clearly intense and dominant. This observation suggests that increased annealing temperature resulted in the formation of larger density of Si NCs due to improved recrystallization and simultaneous reduction in the defect density. The insets of Figs. 4(a) and 4(b) show the photographs of the EL emissions from P900 (blue) and P1000 (yellow), respectively.

EL spectra from the P1060 sample with varying injection current densities at room temperature are shown in Fig. 4(c). The emission range is $440-890 \mathrm{~nm}$ with a single peak centered at $\sim 650 \mathrm{~nm}$. Here, the peak position of the EL emission is also similar to that observed in PL emission from the sample [Fig. 1(d)]. The absence of defect-related peaks confirms that the emission is entirely from quantum-confined $\mathrm{Si}$ NCs. The inset of Fig. 4(c) presents the photograph of red EL emission from the device. Figure 4(d) presents the normalized EL spectra from Si NC samples annealed at different temperatures at an applied forward bias of $30 \mathrm{~V}$. For P900 and P1000 samples, only EL emission peaks from Si NCs are considered. The plot clearly shows a monotonous redshift in the peak position with increasing annealing temperature in agreement with PL results. It may be noted that several results have been reported on the dominance of interference phenomena on the PL peak position of Si NCs due to the thickness-dependent optical-geometrical effects in the superlattice and multilayer sample structures. ${ }^{33-37}$ However, such interference effects are unlikely to be dominant here since the thickness $(\sim 350 \mathrm{~nm})$ and Si content $(\sim 4.8 \%)$ in Si-rich oxide have been kept constant in our study. In order to substantiate this claim, the optical thickness for each sample (P900, 

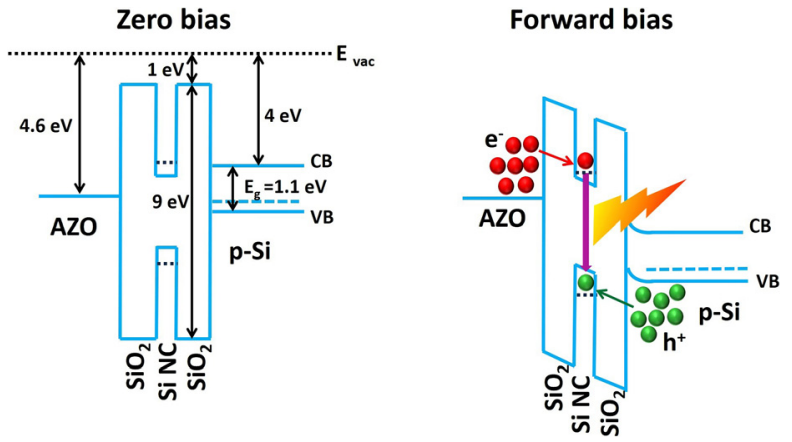

FIG. 5. Schematic band alignment of the fabricated MOSLED using Si NCs embedded into the $\mathrm{SiO}_{2}$ matrix (a) at zero bias and (b) at forward bias conditions depicting the carrier transport and EL emission mechanisms.

P1000, and P1060) has been calculated by estimating the effective refractive index using Bruggeman effective-medium approximation. $^{38,39}$ The calculated optical thicknesses are found to be $547.1 \mathrm{~nm}$, $546.4 \mathrm{~nm}$, and $546.0 \mathrm{~nm}$ for P900, P1000, and P1060 samples, respectively, exhibiting the variation to be very minute. So it is concluded that the interference effect, if any, will nearly be the same in all samples keeping the differential shift unaltered and attributing only to the quantum size effect. Moreover, a close agreement in size-dependent PL and EL emission peaks (Fig. S5 in the supplementary material) corroborates the radiative recombination of confined carriers within $\mathrm{Si}$ NCs randomly embedded into the high-bandgap $\mathrm{SiO}_{2}$ matrix. The plot of integrated EL intensity of the P1060 sample as a function of injected current density in the forward bias condition and comparison of shifts in the peak position of both PL and EL emissions with respect to annealing temperature for P900, P1000, and P1060 samples are presented in the supplementary material.

To understand the carrier injection and recombination mechanisms within the Si NCs responsible for the EL emission, a schematic energy band diagram is proposed. Figures $5(\mathrm{a})$ and $5(\mathrm{~b})$ present the energy band diagram of $\mathrm{Si}$ NCs embedded within the $\mathrm{SiO}_{2}$ matrix at the flatband condition and under an applied forward bias, respectively. The concerned energy gap values are taken from the literature. ${ }^{25,40}$ Si NCs form a quantum well structure bound on both sides by the dielectric barrier of high energy gap $\mathrm{SiO}_{2}$. The quantum confined energy levels in Si NCs vary as a function of size following Brus' equation given by

$$
E=E_{g}+\frac{h^{2}}{8 R^{2}}\left(\frac{1}{m_{e}^{*}}+\frac{1}{m_{h}^{*}}\right)-\frac{1.8 e^{2}}{4 \pi \varepsilon_{0} \varepsilon_{\alpha} R},
$$

where $E_{g}$ is the bulk bandgap of the semiconductor, $R$ is the radius of the nanocrystals, $\varepsilon_{0}$ is the vacuum permittivity, and $\varepsilon_{\alpha}$ is the high-frequency dielectric constant of the semiconductor. ${ }^{41}$ Under a forward bias, carriers are injected into the system from the electrode and the substrate, which tunnel through $\mathrm{SiO}_{2}$ barriers into the quantum well, as shown in Fig. 5(b). Within the quantum well, holes and electrons recombine to emit photons specific to the energy gap in the quantum well.

In summary, we have fabricated MOSLEDs using Si NCs embedded into the $\mathrm{SiO}_{2}$ matrix and studied its size-tunable emission characteristics at room temperature. The PECVD-grown SRO films have been annealed at different temperatures to form Si NCs of different average sizes. Si NCs exhibited PL emissions with a monotonous redshift with the increase in the size due to the quantum confinement of charge carriers. The carrier decay dynamics manifested the radiative recombination of photo-generated charge carriers on the timescale of microseconds. The carrier transport through Si NCs at high and low electric fields is ascribed to the Fowler-Nordheim and direct tunneling mechanisms, respectively. The observed EL emission from the fabricated MOSLEDs exhibits a size-induced blueshift/redshift of the emission peak. The size-tunable characteristics of both PL and EL emission are attributed exclusively to the QCE of charge carriers within the $\mathrm{Si}$ NC quantum well. The results reveal Si NCs to be potential candidates for CMOS compatible integrated optical sources for future photonic integrated chips.

See the supplementary material for experimental methods, detailed material characterization, ultrafast reflectivity measurement data, and extended EL data and their detailed discussions.

Partial financial support from DST-Meity supported NNetRA "SWI" Project Grant No. DST/NM/NNETRA/2018(G) and MHRD-DRDO IMPRINT “USR” Project Grant No. 3-18/2015T.S.-I (Vol. IV) is gratefully acknowledged. The authors also acknowledge the use of the Ultrafast Spectroscopy Facility of the Department of Physics, IIT Kharagpur.

\section{DATA AVAILABILITY}

The data that support the findings of this study are available within this article and its supplementary material.

\section{REFERENCES}

${ }^{1}$ C. Huh, K. H. Kim, B. K. Kim, W. Kim, H. Ko, C. J. Choi, and G. Y. Sung, Adv. Mater. 22, 5058 (2010).

${ }^{2}$ S. Kim, D. H. Shin, and S. H. Choi, Appl. Phys. Lett. 100, 253103 (2012).

${ }^{3}$ F. Priolo, T. Gregorkiewicz, M. Galli, and T. F. Krauss, Nat. Nanotechnol. 9, 19 (2014).

${ }^{4}$ S. Singh, A. K. Katiyar, A. Sarkar, P. K. Shihabudeen, A. R. Chaudhuri, D. K. Goswami, and S. K. Ray, Nanotechnology 31, 115206 (2020).

${ }^{5}$ S. K. Ray, S. Maikap, W. Banerjee, and S. Das, J. Phys. D: Appl. Phys. 46, 153001 (2013).

${ }^{6}$ S.-W. Fu, H.-J. Chen, H.-T. Wu, S.-P. Chen, and C.-F. Shih, Nanoscale 8, 7155 (2016).

${ }^{7}$ G. Ledoux, J. Gong, F. Huisken, O. Guillois, and C. Reynaud, Appl. Phys. Lett. 80, 4834 (2002).

${ }^{8}$ S. Takeoka, M. Fujii, and S. Hayashi, Phys. Rev. B 62, 16820 (2000).

${ }^{9}$ A. K. Katiyar, A. K. Sinha, S. Manna, and S. K. Ray, ACS Appl. Mater. Interfaces 6, 15007 (2014).

${ }^{10}$ L. T. Canham, Appl. Phys. Lett. 57, 1046 (1990).

${ }^{17}$ S. K. Ray, A. K. Katiyar, and A. K. Raychaudhuri, Nanotechnology 28, 092001 (2017).

${ }^{12}$ L. T. Canham, W. Y. Leong, M. I. J. Beale, T. I. Cox, and L. Taylor, Appl. Phys. Lett. 61, 2563 (1992).

${ }^{13}$ A. Sarkar, A. K. Katiyar, S. Mukherjee, S. Singh, S. K. Singh, A. K. Das, and S. K. Ray, ACS Appl. Electron. Mater. 1, 25 (2019).

${ }^{14}$ A. Sarkar, S. Mukherjee, A. K. Das, and S. K. Ray, Nanotechnology 30, 485202 (2019).

15. Wu, J. Dai, Y. Shao, and Y. Sun, RSC Adv. 5, 83581 (2015).

${ }^{16}$ D. P. Puzzo, E. J. Henderson, M. G. Helander, Z. Wang, G. A. Ozin, and Z. Lu, Nano Lett. 11, 1585 (2011).

${ }^{17}$ S. Lombardo, S. Coffa, C. Bongiorno, C. Spinella, E. Castagna, A. Sciuto, C. Gerardi, F. Ferrari, B. Fazio, and S. Privitera, Mater. Sci. Eng., B 69-70, 295 (2000). 
${ }^{18}$ G. Franzò, A. Irrera, E. C. Moreira, M. Miritello, F. Iacona, D. Sanfilippo, G. Di Stefano, P. G. Fallica, and F. Priolo, Appl. Phys. A 74, 1 (2002).

${ }^{19}$ W. D. A. M. De Boer, D. Timmerman, K. Dohnalová, I. N. Yassievich, H. Zhang, W. J. Buma, and T. Gregorkiewicz, Nat. Nanotechnol. 5, 878 (2010).

${ }^{20}$ Y. Kanemitsu, T. Ogawa, K. Shiraishi, and K. Takeda, Phys. Rev. B 48, 4883 (1993).

${ }^{21}$ J. P. Wilcoxon and G. A. Samara, Appl. Phys. Lett. 74, 3164 (1999).

${ }^{22}$ F. Iacona, G. Franzò, and C. Spinella, J. Appl. Phys. 87, 1295 (2000).

${ }^{23}$ A. A. G. Fernández, M. A. Mijares, A. M. Sánchez, and K. M. Leyva, J. Appl. Phys. 108, 043105 (2010).

${ }^{24}$ O. Jambois, B. Garrido, P. Pellegrino, J. Carreras, A. Ṕrez-Rodríguez, J. Montserrat, C. Bonafos, G. Benassayag, and S. Schamm, Appl. Phys. Lett. 89, 253124 (2006).

${ }^{25}$ D. Di, I. Perez-Wurfl, L. Wu, Y. Huang, A. Marconi, A. Tengattini, A. Anopchenko, L. Pavesi, and G. Conibeer, Appl. Phys. Lett. 99, 251113 (2011).

${ }^{26}$ A. Morales-Sánchez, J. Barreto, C. Domínguez, M. Aceves-Mijares, M. Perálvarez, B. Garrido, and J. A. Luna-López, Nanotechnology 21, 085710 (2010).

${ }^{27}$ J. López-Vidrier, Y. Berencén, S. Hernández, B. Mundet, S. Gutsch, J. Laube, D. Hiller, P. Löper, M. Schnabel, S. Janz, M. Zacharias, and B. Garrido, Nanotechnology 26, 185704 (2015).

${ }^{28}$ G. F. Grom, D. J. Lockwood, J. P. McCaffrey, H. J. Labbé, P. M. Fauchet, B. White, J. Diener, D. Kovalev, F. Koch, and L. Tsybeskov, Nature 407, 358 (2000).

${ }^{29}$ D. Riabinina, C. Durand, J. Margot, M. Chaker, G. A. Botton, and F. Rosei, Phys. Rev. B 74, 075334 (2006).
${ }^{30}$ G. R. Lin, C. J. Lin, C. K. Lin, L. J. Chou, and Y. L. Chueh, J. Appl. Phys. 97, 094306 (2005).

${ }^{31}$ G.-R. Lin, C.-J. Lin, and C. Lin, Opt. Express 15, 2555 (2007).

${ }^{32}$ A. Anopchenko, A. Marconi, M. Wang, G. Pucker, P. Bellutti, and L. Pavesi, Appl. Phys. Lett. 99, 181108 (2011).

${ }^{33}$ D. Hiller, A. Zelenina, S. Gutsch, S. A. Dyakov, L. López-Conesa, J. LópezVidrier, S. Estradé, F. Peiró, B. Garrido, J. Valenta, M. Kořínek, F. Trojánek, P. Malý, M. Schnabel, C. Weiss, S. Janz, and M. Zacharias, J. Appl. Phys. 115, 204301 (2014).

${ }^{34}$ M. Schnabel, C. Summonte, S. A. Dyakov, M. Canino, L. López-Conesa, P. Löper, S. Janz, and P. R. Wilshaw, J. Appl. Phys. 117, 045307 (2015).

${ }^{35}$ A. Zelenina, S. A. Dyakov, D. Hiller, S. Gutsch, V. Trouillet, M. Bruns, S. Mirabella, P. Löper, L. López-Conesa, J. López-Vidrier, S. Estradé, F. Peiró, B. Garrido, J. Bläsing, A. Krost, D. M. Zhigunov, and M. Zacharias, J. Appl. Phys. 114, 184311 (2013).

${ }^{36}$ S. A. Dyakov, D. M. Zhigunov, A. Hartel, M. Zacharias, T. S. Perova, and V. Y. Timoshenko, Appl. Phys. Lett. 100, 061908 (2012).

${ }^{37}$ R. Ferre, B. Garrido, P. Pellegrino, M. Perálvarez, C. García, J. A. Moreno, J. Carreras, and J. R. Morante, J. Appl, Phys. 98, 084319 (2005).

${ }^{38}$ J. A. Moreno, B. Garrido, P. Pellegrino, C. Garcia, J. Arbiol, J. R. Morante, P. Marie, F. Gourbilleau, and R. Rizk, J. Appl. Phys. 98, 013523 (2005).

${ }^{39}$ G. Vijaya prakash, M. Cazzanelli, Z. Gaburro, L. Pavesi, F. Iacona, G. Franzò, and F. Priolo, J. Mod. Opt. 49, 719-730 (2002).

${ }^{40}$ G.-R. Lin, Y. Pai, C. Lin, and C. Chen, Appl. Phys. Lett. 96, 263514 (2010).

${ }^{41}$ L. Brus, IEEE J. Quantum Electron. 22, 1909 (1986). 